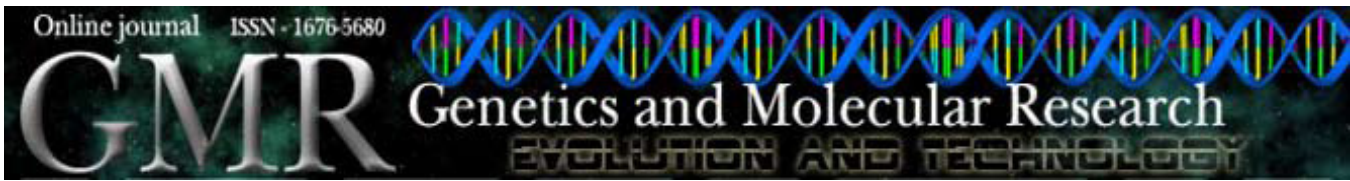

\title{
$\beta 3$-adrenergic receptor polymorphism is related to cardiometabolic risk factors in obese Brazilian subjects
}

\author{
V.A. Genelhu ${ }^{1}$, E.A. Francischetti ${ }^{1}$, S.F.P. Duarte ${ }^{1}$, B.M.J. Celoria ${ }^{1}$, \\ R.C. Oliveira ${ }^{1}$, P.H. Cabello ${ }^{3}$ and M.M.G. Pimentel ${ }^{2}$ \\ ${ }^{1}$ Laboratório de Fisiopatologia Clínica e Experimental (CLINEX), \\ Clínica de Hipertensão, Faculdade de Ciências Médicas, \\ Universidade do Estado do Rio de Janeiro, Rio de Janeiro, RJ, Brasil \\ ${ }^{2}$ Departamento de Genética, Instituto de Biologia Roberto Alcântara Gomes, \\ Serviço de Génetica Humana, Universidade do Estado do Rio de Janeiro, \\ Rio de Janeiro, RJ, Brasil \\ ${ }^{3}$ Laboratório de Genética Humana, Instituto Oswaldo Cruz, \\ Ministério da Saúde, Rio de Janeiro, RJ, Brasil \\ Corresponding author: E.A. Francischetti \\ E-mail: francischetti@globo.com
}

Genet. Mol. Res. 9 (3): 1392-1397 (2010)

Received March 11, 2010

Accepted May 20, 2010

Published July 20, 2010

DOI 10.4238/vol9-3gmr819

\begin{abstract}
We determined whether $A D R \beta 3$ polymorphism is associated with obesity-related traits in 140 obese patients. Molecular analysis was performed by PCR and RFLP. Individuals carrying the Arg64 allele had a lower waist-to-hip ratio, higher adiponectin and high-density lipoprotein cholesterol levels, and a tendency towards lower blood pressure. In contrast, Trp64/Trp64 carriers were at greater risk for dysmetabolic phenotypes (odds ratio $=2.88, \mathrm{P}=0.03$ ).
\end{abstract}

Key words: Adrenergic receptor; Obesity; Polymorphism; Adiponectin; Cardiometabolic risk factor 


\section{INTRODUCTION}

The sympathetic nervous system regulates energy balance through thermogenesis, and the $\beta 3$-adrenergic receptor system is important in mediating lipolysis stimulation by catecholamines, as well as in the development of obesity (Clement et al., 1995)

Some studies have reported that the Trp64Arg polymorphism of the $\beta 3$-adrenergic receptor gene $(A D R \beta 3)$ can be associated with several conditions such as higher incidence of abdominal obesity (Kim-Motoyama et al., 1997), insulin resistance and type 2 diabetes (Morcillo et al., 2008). However, these findings have not been confirmed in other studies (Gagnon et al., 1996). Simultaneously, obesity and hypertension have been considered related to polymorphisms of the $A D R \beta 3$ gene, but the reports have been restricted to Asian people (Masuo et al., 2005).

The present study was conducted to determine in a group of obese Brazilian subjects of multiethnic origin whether the Trp64Arg polymorphism of the $A D R \beta 3$ gene is associated with a significant effect on features related to metabolic syndrome and to adiponectin levels, an adipocyte-derived collagen-like protein, considered a key factor of metabolic syndrome (Patel et al., 2006)

\section{MATERIAL AND METHODS}

\section{Study population}

This was a cross-sectional study. A total of 209 individuals, aged 18 to 71 years, were selected among the patients of the Hypertension Clinic (CLINEX) of the Rio de Janeiro State University, who were part of an ongoing study on the mechanistic and genetic aspects of blood pressure variation in overweight and obese individuals. It is worth noting that the Rio de Janeiro State population is peculiar due to its multiethnic admixture characterized by Caucasians, African descendants and Mulattos (people with a known family history of admixture between African-descendants and Caucasian populations).

Stable weight for at least three months was required. The patients underwent routine blood chemistries, urinalysis, electrocardiography, echocardiography, duplex Doppler ultrasonography of renal arteries, and endocrine evaluation. Individuals with severe forms of hypertension, and those with any evidence of complications from endocrine, metabolic, cardiovascular, and cerebrovascular diseases were excluded.

One hundred and forty individuals [ 41 men and 99 women, mean age of $45.2 \pm 12.4$ years, body mass index $\left.(\mathrm{BMI})=38.5 \pm 8.1 \mathrm{~kg} / \mathrm{m}^{2}\right]$ comprised the study sample. All subjects included in the study were instructed to stop using any medication that could affect blood pressure levels, insulin sensitivity, and serum lipid concentration at least two weeks before entering the study. Before the study, written informed consent was obtained from each participant.

The study protocol was approved by the Committee on Ethics and Research of the Pedro Ernesto University Hospital of the Rio de Janeiro State University.

\section{Clinical features}

Blood pressure was recorded using a Dinamap 1846 Critikon automated sphygmoma- 
nometer after a resting period of at least $10 \mathrm{~min}$ in the sitting position.

An appropriate arm cuff was used. The mean of the last four readings was used in the analysis. Waist-to-hip ratio was used as an indicator of central adiposity. Metabolic syndrome was defined according to criteria of the Third Report of the National Cholesterol Education Program (Grundy et al., 2005). Blood samples were collected after a 12-h fasting period and were stored at $-80^{\circ} \mathrm{C}$ for subsequent radioimmunoassay, which was used to determine plasma adiponectin and insulin (Linco Research, St. Charles, MO, USA - double-antibody solid-phase enzyme immunoassay). The intra- and interassay coefficients of variation for insulin were 4.4 and $6.0 \%$, respectively. The intra- and interassay coefficients of variation for adiponectin were 3.9 and $8.5 \%$, respectively. The insulin resistance status was assessed by use of the homeostasis model assessment of insulin resistance (HOMA-IR) index, i.e., serum insulin [ $\mu \mathrm{U} /$ $\mathrm{mL}]$ x plasma glucose $[\mathrm{mM}] / 22.5$. Serum lipid profiles were measured by use of enzymatic methods.

\section{Molecular analysis}

Genomic DNA was obtained from peripheral blood leukocytes using the salting-out method. Polymorphism was genotyped by use of the polymerase chain reaction-restriction fragment length polymorphism (PCR-RFLP) assay with the previously described primer pairs (Clement et al., 1995). PCR was performed on total volumes of $25 \mu \mathrm{L}$, using $100 \mathrm{ng}$ DNA, 1X PCR buffer, $2.0 \mathrm{mM} \mathrm{MgCl}, 0.25 \mathrm{mM}$ of each dNTP, 2.5 pmol of each primer, and $1 \mathrm{U}$ Taq DNA polymerase. DNA was denatured for $5 \mathrm{~min}$ at $95^{\circ} \mathrm{C}$, and amplified for 40 cycles with annealing temperature of $66^{\circ} \mathrm{C}$. Ten microliters of the PCR products was digested with $2.5 \mathrm{U}$ of the restriction endonuclease Bst $\mathrm{NI}$ (New England Biolabs, Inc.) for $4 \mathrm{~h}$ at $60^{\circ} \mathrm{C}$ and analyzed by electrophoresis on $8 \%$ polyacrylamide gels stained with silver nitrate.

\section{Statistical analysis}

Data are reported as means $\pm \mathrm{SD}$. A P value $<0.05$ was considered to be statistically significant and was adjusted for covariates when appropriate. Values of triglycerides, insulin, adiponectin, and HOMA-IR were log-transformed to improve normality. Data comparisons were carried out with the Student $t$-test, controlling for gender and age. Categorical variables were compared across the three groups with the chi-square test.

Relationships among variables were computed by use of Pearson correlation coefficients. A logistic regression analysis adjusted for age, gender, waist circumference, triglycerides, blood pressure, glucose, and high-density lipoprotein cholesterol (HDL-C) was carried out to assess the potential risk of the metabolic syndrome phenotype, evaluated as a categorical variable, according to the $A D R \beta 3$ polymorphism. Analysis was performed for combined Trp64/Arg64 and Arg64/Arg64 as the group with Arg64 allele and for Trp64/Trp64 as the group without the Arg64 variant. All statistical analyses were performed with SPSS, version 12.0 (Lead Technologies Station, Chicago, USA).

\section{RESULTS}

Table 1 shows the distribution of variables according to the $A D R \beta 3$ genotypes. 


\begin{tabular}{|c|c|c|c|}
\hline \multirow[t]{2}{*}{ Variables } & \multicolumn{3}{|c|}{ Genotypes } \\
\hline & $\begin{array}{c}\text { Trp64/Trp64 } \\
(\mathrm{N}=120)\end{array}$ & $\begin{array}{c}\text { Trp64/Arg64 + Arg64/Arg64 } \\
(\mathrm{N}=20)\end{array}$ & $\mathrm{P}$ \\
\hline Age (years) & $45.5 \pm 12.1$ & $46.3 \pm 13.1$ & 0.75 \\
\hline Gender (female/male) & $82 / 38$ & $17 / 3$ & 0.13 \\
\hline Body mass index $\left(\mathrm{kg} / \mathrm{m}^{2}\right)$ & $38.1 \pm 8.1$ & $40.8 \pm 7.2$ & 0.17 \\
\hline Waist circumference $(\mathrm{cm})$ & $112.2 \pm 15.1$ & $113.7 \pm 14.6$ & 0.68 \\
\hline Hip circumference (cm) & $119.1 \pm 14.5$ & $128.2 \pm 19.0$ & $0.01 / 0.01 *$ \\
\hline Waist-to-hip ratio & $0.95 \pm 0.10$ & $0.89 \pm 0.10$ & $0.03 / 0.04 *$ \\
\hline Total cholesterol (mg/dL) & $202.4 \pm 42.8$ & $200.0 \pm 41.2$ & 0.81 \\
\hline LDL cholesterol (mg/dL) & $127.0 \pm 36.1$ & $118.5 \pm 31.8$ & 0.32 \\
\hline HDL cholesterol (mg/dL) & $44.8 \pm 10.0$ & $49.5 \pm 11.3$ & $0.04 / 0.04 *$ \\
\hline Glucose (mg/dL) & $106.5 \pm 27.4$ & $101.6 \pm 17.8$ & 0.44 \\
\hline Triglycerides (mg/dL) & $152.8 \pm 83.6$ & $159.8 \pm 82.2$ & 0.67 \\
\hline Insulin $(\mathrm{mU} / \mathrm{L})$ & $24.1 \pm 12.4$ & $20.5 \pm 7.8$ & 0.44 \\
\hline HOMA-IR & $6.4 \pm 3.9$ & $5.3 \pm 2.7$ & 0.40 \\
\hline Adiponectin $(\mu \mathrm{g} / \mathrm{mL})$ & $6.9 \pm 4.5$ & $8.1 \pm 3.5$ & $0.03 / 0.04 *$ \\
\hline Systolic blood pressure $(\mathrm{mmHg})$ & $131 \pm 17$ & $125 \pm 14$ & 0.13 \\
\hline Diastolic blood pressure $(\mathrm{mmHg})$ & $84 \pm 11$ & $79 \pm 13$ & 0.09 \\
\hline Mean arterial pressure $(\mathrm{mmHg})$ & $100 \pm 12$ & $94 \pm 13$ & 0.07 \\
\hline Uric acid $(\mathrm{mg} / \mathrm{dL})$ & $5.6 \pm 1.6$ & $5.3 \pm 1.5$ & 0.50 \\
\hline Creatinine (mg/dL) & $0.8 \pm 0.2$ & $0.8 \pm 0.1$ & 0.89 \\
\hline
\end{tabular}

*Adjusted for age gender. $\mathrm{LDL}=$ low-density lipoprotein; HDL = high-density lipoprotein; HOMA-IR = homeostasis model assessment of insulin resistance.

Genotypic distribution satisfied Hardy-Weinberg equilibrium $\left(\chi^{2}=1.743 ; \mathrm{P}=0.41\right)$.

One hundred and twenty (85.7\%) subjects were classified as homozygous [Trp64/ Trp64] and 20 (14.3\%) individuals as Arg64-allele carriers [Trp64/Arg64 and Arg64/Arg64]. The Arg64-allele carriers showed significantly higher values of plasma adiponectin, HDL-C and hip circumference, while the waist-to-hip ratio was significantly lower compared with that of the group without the Arg64 allele. These results remained significant after adjustment for age and gender. The Arg64-allele carriers also showed lower levels of diastolic blood pressure and mean arterial pressure when compared with the Trp/Trp individuals, albeit not significant $(\mathrm{P}=0.09$ and 0.07 , respectively).

In the subjects with the Trp64/Trp64 genotype, HOMA-IR was significantly and positively correlated with BMI, waist-to-hip ratio, systolic blood pressure, diastolic blood pressure, and plasma triglycerides $(\mathrm{r}=0.37,0.32,0.29,0.27$, and $0.32, \mathrm{P}<0.01$, respectively).

In the Arg64-allele carriers, plasma adiponectin exhibited a negative correlation with waist-to-hip ratio $(\mathrm{r}=-0.51, \mathrm{P}=0.009)$. A logistic regression analysis to assess the potential risk for metabolic syndrome showed that individuals without the Arg64 variant had a greater risk for dysmetabolic phenotypes $(\mathrm{OR}=2.88, \mathrm{P}=0.03)($ Table 2$)$.

Table 2. Logistic regression analysis for metabolic syndrome.

\begin{tabular}{|c|c|c|c|c|c|c|}
\hline & \multicolumn{2}{|c|}{ Frequency distribution } & \multicolumn{4}{|c|}{ Logistic models } \\
\hline & Trp64/Trp64 & Trp64/Arg64 + Arg64/Arg64 & $P$ & Odds ratio & $95 \% \mathrm{CI}$ & $P$ \\
\hline $\begin{array}{l}\text { With MetSynd } \\
\text { Without MetSynd }\end{array}$ & $\begin{array}{l}47(39.2 \%) \\
73(60.8 \%)\end{array}$ & $\begin{array}{r}7(35 \%) \\
13(65 \%)\end{array}$ & 0.049 & 2.88 & $(1.07-7.75)$ & 0.03 \\
\hline
\end{tabular}

The logistic regression model was adjusted for age, gender, waist circumference, triglycerides, blood pressure, glucose, and HDL cholesterol. MetSynd = metabolic syndrome. 


\section{DISCUSSION}

In the most general sense, the results presented demonstrate that individuals carrying the Arg64 allele had a distinct benign metabolic and hemodynamic phenotype of obesity, when compared with subjects homozygous for the Trp64 allele, who exhibited a mark risk for dysmetabolic phenotypes.

There are few reports on the genetic relationship of the Trp64Arg polymorphism with hypertension in obesity (Fujisawa et al., 1997; Melis et al., 2002; Masuo et al., 2005). Fugisawa et al. (1997) showed that the Arg64-allele frequency in hypertensive subjects was similar to that in normotensive subjects. More recently, Masuo et al. (2005) observed no association of the $\beta 3$-adrenergic receptor genotypes with weight gain-related blood pressure.

In the present study, the Arg64-allele carriers had higher values of adiponectin, HDL-C and hip circumference, and a lower waist-to-hip ratio. They also exhibited the lower-body, glutealfemoral form of obesity, known as "gynoid" obesity, suggesting that a metabolically benign obesity may be identified genetically. In addition, a trend towards a lower blood pressure levels was also observed in the Arg64-allele carriers, which can be partially attributed to high adiponectin levels. In the Bogalusa Heart Study, individuals with higher values of plasma adiponectin showed the lowest blood pressure levels (Patel et al., 2006). These observations are in agreement with those by Vague (1956) who, 50 years ago, first described an important heterogeneity among his obese patients, and distinguished "gynoid" obesity from the "android" or central abdominal form of obesity, which ends up associated with hypertension, insulin resistance and lipid abnormalities.

The abnormalities of central obesity have now become widely known as metabolic syndrome (Grundy et al., 2005). Interestingly, a logistic regression analysis of the metabolic syndrome features of our patients confirmed that Trp64/Trp64 subjects, unlike Arg64-allele carriers, were characterized by an insulin-resistant state, exhibiting elevated fasting plasma insulin and HOMA-IR levels that correlated significantly and directly with BMI, waist-to-hip ratio and blood pressure in those individuals.

A major limitation of the present report is the small sample size, with only 20 subjects carrying the Arg allele. Thus, a prospective study with a larger population would be required to confirm our data and to assess a cause-effect relationship between blood pressure variation and cardiometabolic risk factors with the Trp64Arg polymorphism of $A D R \beta 3$. In addition, the study was limited to obese multiethnic subjects originating from a university referral center, most of them clustering several cardiometabolic risk factors.

Thus, we do not know if the findings can be extended to populations including apparently healthier obese individuals or different ethnic mixtures. Finally, there were more females (85\%) in the Arg64 carriers compared to the Trp64/Trp64 group (68\%). This may in itself explain the more "gynoid" proportions of the Arg64 carriers, although data were adjusted for gender.

In conclusion, in this ethnically mixed population sample, the Arg-allele carriers of the $\beta 3$-adrenergic receptor gene had a benign phenotype of obesity when compared with the Trp64/Trp64 subjects. This assumption was based on a significantly higher concentration of plasma adiponectin and HDL-C, and the "gynoid" body fat distribution.

\section{REFERENCES}

Clement K, Vaisse C, Manning BS, Basdevant A, et al. (1995). Genetic variation in the $\beta 3$-adrenergic receptor and an increased capacity to gain weight in patients with morbid obesity. N. Engl. J. Med. 333: 352-354. 
Fujisawa T, Ikegami H, Yamato E, Hamada Y, et al. (1997). Trp64Arg mutation of $\beta 3$-adrenergic receptor in essential hypertension: insulin resistance and the adrenergic system. Am. J. Hypertens. 10: 101-105.

Gagnon J, Mauriege P, Roy S, Sjostrom D, et al. (1996). The Trp64Arg mutation of the beta3 adrenergic receptor gene has no effect on obesity phenotypes in the Quebec Family Study and Swedish Obese Subjects cohorts. J. Clin. Invest. 98: 2086-2093.

Grundy SM, Cleeman JI, Daniels SR, Donato KA, et al. (2005). Diagnosis and management of the metabolic syndrome: an American Heart Association/National Heart, Lung, and Blood Institute Scientific Statement. Circulation 112: $2735-2752$.

Kim-Motoyama H, Yasuda K, Yamaguchi T, Yamada N, et al. (1997). A mutation of the beta 3-adrenergic receptor is associated with visceral obesity but decreased serum triglyceride. Diabetologia 40: 469-472.

Masuo K, Katsuya T, Fu Y, Rakugi H, et al. (2005). Beta2- and beta3-adrenergic receptor polymorphisms are related to the onset of weight gain and blood pressure elevation over 5 years. Circulation 111:3429-3434.

Melis MG, Secchi G, Brizzi P, Severino C, et al. (2002). The Trp64Arg beta3-adrenergic receptor amino acid variant confers increased sensitivity to the pressor effects of noradrenaline in Sardinian subjects. Clin. Sci. 103: 397-402.

Morcillo S, Cardona F, Rojo-Martinez G, Almaraz MC, et al. (2008). Effect of the combination of the variants -75G/A APOA1 and Trp64Arg ADRB3 on the risk of type 2 diabetes (DM2). Clin. Endocrinol. 68: 102-107.

Patel DA, Srinivasan SR, Xu JH, Chen W, et al. (2006). Adiponectin and its correlates of cardiovascular risk in young adults: the Bogalusa Heart Study. Metabolism 55: 1551-1557.

Vague J (1956). The degree of masculine differentiation of obesities: a factor determining predisposition to diabetes, atherosclerosis, gout, and uric calculous disease. Am. J. Clin. Nutr. 4: 20-34. 